TITLE:

\title{
Blue photoluminescence and Auger recombination of carriers in SrTiO3 nanoparticles
}

\section{$\operatorname{AUTHOR}(\mathrm{S}):$}

Yamada, Yasuhiro; Suzuki, Keigo; Kanemitsu, Yoshihiko

\section{CITATION:}

Yamada, Yasuhiro ... [et al]. Blue photoluminescence and Auger recombination of carriers in SrTiO3 nanoparticles. Applied Physics Letters 2011, 99(9): 093101.

\section{ISSUE DATE:}

2011-08-30

URL:

http://hdl.handle.net/2433/159452

RIGHT:

(C) 2011 American Institute of Physics 


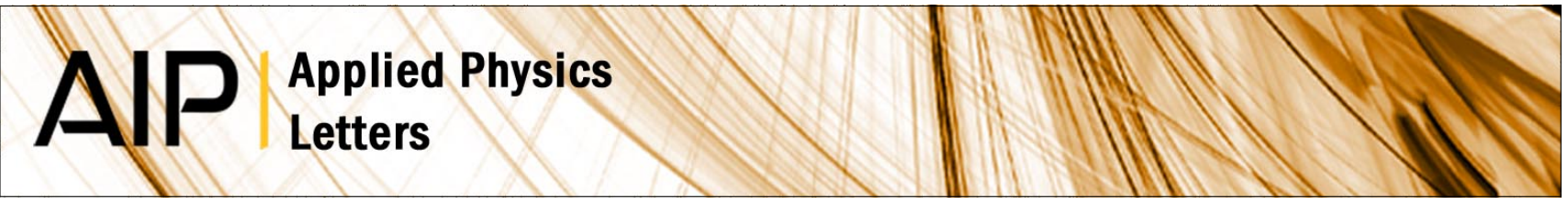

\section{Blue photoluminescence and Auger recombination of carriers in SrTiO3 nanoparticles}

Yasuhiro Yamada, Keigo Suzuki, and Yoshihiko Kanemitsu

Citation: Appl. Phys. Lett. 99, 093101 (2011); doi: 10.1063/1.3626594

View online: http://dx.doi.org/10.1063/1.3626594

View Table of Contents: http://apl.aip.org/resource/1/APPLAB/v99/i9

Published by the American Institute of Physics.

\section{Related Articles}

Photoluminescence spectroscopy and energy-level analysis of metal-organic-deposited Ga2O3:Cr3+ films J. Appl. Phys. 112, 063522 (2012)

Intense green and red upconversion emission of Er3+,Yb3+ co-doped CaZrO3 obtained by a solution combustion reaction

J. Appl. Phys. 112, 063105 (2012)

Intense upconversion luminescence and origin study in Tm3+/Yb3+ codoped calcium scandate Appl. Phys. Lett. 101, 121905 (2012)

Photoluminescence dynamics in solid formulations of colloidal PbSe quantum dots: Three-dimensional versus two-dimensional films

Appl. Phys. Lett. 101, 121904 (2012)

Formation probability of $\mathrm{Cr}$-Nd pair and energy transfer from $\mathrm{Cr}$ to Nd in $\mathrm{Y} 3 \mathrm{Al} 5 \mathrm{O} 12$ ceramics codoped with $\mathrm{Nd}$ and $\mathrm{Cr}$

J. Appl. Phys. 112, 063508 (2012)

\section{Additional information on Appl. Phys. Lett.}

Journal Homepage: http://apl.aip.org/

Journal Information: http://apl.aip.org/about/about_the_journal

Top downloads: http://apl.aip.org/features/most_downloaded

Information for Authors: http://apl.aip.org/authors

\section{ADVERTISEMENT}
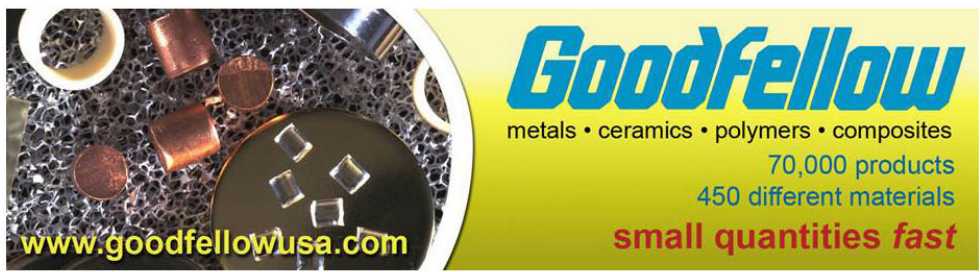


\title{
Blue photoluminescence and Auger recombination of carriers in $\mathrm{SrTiO}_{3}$ nanoparticles
}

\author{
Yasuhiro Yamada, ${ }^{1}$ Keigo Suzuki, ${ }^{2}$ and Yoshihiko Kanemitsu ${ }^{1,3, a)}$ \\ ${ }^{1}$ Institute for Chemical Research, Kyoto University, Uji, Kyoto 611-0011, Japan \\ ${ }^{2}$ Murata Manufacturing Co Ltd., Nagaokakyo-shi, Kyoto 617-8555, Japan \\ ${ }^{3}$ Photonics and Electronics Science and Engineering Center, Kyoto University, Kyoto 615-8510, Japan
}

(Received 29 April 2011; accepted 30 July 2011; published online 30 August 2011)

\begin{abstract}
We studied photoluminescence (PL) spectrum and dynamics of $\mathrm{SrTiO}_{3}$ nanoparticles. A broad blue PL band is observed at around $2.9 \mathrm{eV}$ in nanoparticle thin films. The excitation-density dependence of the blue PL intensity indicates that nonradiative Auger recombination dominates the carrier recombination process under strong photoexcitation. The intrinsic Auger recombination rate in $\mathrm{SrTiO}_{3}$ nanoparticles is much larger than that of $\mathrm{SrTiO}_{3}$ bulk crystals. The origin of enhanced Auger recombination in $\mathrm{SrTiO}_{3}$ nanoparticles is discussed. (c) 2011 American Institute of Physics. [doi:10.1063/1.3626594]
\end{abstract}

Recently, perovskite oxides have attracted a great deal of attention as unique device materials because they have a wide variety of fascinating and multifunctional properties. $\mathrm{SrTiO}_{3}$ is one of the most promising oxides because of its high dielectric constant, high electron mobility, and advanced optical properties. ${ }^{1-5} \mathrm{SrTiO}_{3}$ is a standard substrate material for oxide thin film growth and, thus, it becomes a platform for future oxide-based electronics. $\mathrm{SrTiO}_{3}$ is also an indirect-gap semiconductor, ${ }^{6,7}$ and its band-gap energy is $3.245 \mathrm{eV}^{7}$ Strongly photoexcited and electron-doped $\mathrm{SrTiO}_{3}$ crystals show blue photoluminescence (PL) at around $2.9 \mathrm{eV}$ at room temperature ${ }^{8,9}$ and band-to-band PL at around 3.2 $\mathrm{eV}$ at low temperatures. ${ }^{7}$ The interest to $\mathrm{SrTiO}_{3}$ is further prompted by observation of unique electronic properties of two-dimensional electron gas systems at $\mathrm{SrTiO}_{3} / \mathrm{LaAlO}_{3}$ heterointerfaces ${ }^{10}$ delta-doped $\mathrm{SrTiO}_{3}$ layer, ${ }^{11}$ and bare $\mathrm{SrTiO}_{3}$ surfaces. ${ }^{12,13}$ These $\mathrm{SrTiO}_{3}$ interfaces and surfaces show a number of appealing properties such as high electron mobility, superconductivity, and magnetoresistance. ${ }^{10-15}$ Moreover, the surface of $\mathrm{SrTiO}_{3}$ crystals plays an important role in optical properties: the oxygen-deficient surface layer exists in $\mathrm{SrTiO}_{3}$ bulk crystals and causes efficient PL from the surface metallic layer. ${ }^{12}$ Since their surface effects are more pronounced due to large surface-to-volume ratios, nanoparticles provide unique optical properties, which are not observed in bulk crystals. For example, oxidized surfaces of indirect-gap Si and Ge semiconductor nanoparticles show efficient room-temperature PL. ${ }^{16,17}$ Thus, it is anticipated that $\mathrm{SrTiO}_{3}$ nanoparticles would show attractive PL properties, different from those of bulk crystals. However, the PL properties of $\mathrm{SrTiO}_{3}$ nanoparticles are not clear.

In this letter, we studied PL spectrum and dynamics of $\mathrm{SrTiO}_{3}$ nanoparticles. We found a broad blue PL band in $\mathrm{SrTiO}_{3}$ nanoparticles under strong pulse laser excitation. The excitation-density dependence of the blue PL intensity indicates that carrier recombination dynamics is dominated by three-carrier Auger recombination. The Auger recombination rate of $\mathrm{SrTiO}_{3}$ nanoparticles is much larger than that of

\footnotetext{
${ }^{a)}$ Electronic mail: kanemitu@scl.kyoto-u.ac.jp.
}

bulk crystals. We discuss surface effects on the Auger recombination rate in $\mathrm{SrTiO}_{3}$ nanoparticles.

The $\mathrm{SrTiO}_{3}$ nanoparticles were synthesized using the microemulsion method, similar to previous reports. ${ }^{18,19}$ The microemulsion that consisted of small water droplets in hydrophobic solvent was prepared using a cyclohexane/NP$10 /$ water system. Strontium iso-propoxide $\left(\mathrm{Sr}\left(\mathrm{OiC}_{3} \mathrm{H}_{7}\right)_{2}\right)$ and titanium iso-propoxide $\left(\mathrm{Ti}\left(\mathrm{OiC}_{3} \mathrm{H}_{7}\right)_{4}\right)$ were dissolved with 2-methoxyethanol and added to the miscroemulsion. The hydrolysis reaction of metal alkoxides took place in the water droplets by stirring the mixed solutions for a week, and eventually a clear emulsion of nanocrystalline $\mathrm{SrTiO}_{3}$ particles was obtained. $\mathrm{SrTiO}_{3}$ nanoparticles were deposited on a Pt/ sapphire substrate using a spin-coating method at $4000 \mathrm{rpm}$ for $30 \mathrm{~s}$. The films were annealed in atmosphere at $150^{\circ} \mathrm{C}$ for $1 \mathrm{~min}$ and then $480^{\circ} \mathrm{C}$ for $3 \mathrm{~min}$. The spin-coating and annealing procedures were repeated for 20 times. Finally, the samples were treated with rapid thermal annealing in atmosphere at different temperatures $\left(550,650\right.$, and $\left.750^{\circ} \mathrm{C}\right)$ for $20 \mathrm{~min}$. Hereafter, these samples are referred to as ST-550, ST-650, and ST-750, respectively.

Time-integrated PL spectra were measured using a monochromator connected to a charge-coupled device camera for detection. We used a continuous-wave (cw) He-Cd laser and a pulse laser (an optical parametric amplifier with $180 \mathrm{fs}$ pulse duration, $200 \mathrm{kHz}$ repetition rate, and $3.82 \mathrm{eV}$ photon energy). The PL decay profiles under pulse laser excitation were measured with a time resolution of about $40 \mathrm{ps}$ using a streak camera and a monochromator. All measurements were carried out at room temperature.

Figure 1(a) shows X-ray diffraction (XRD) patterns of the ST-550 sample and the Pt/sapphire substrate, indicating the monophase cubic structure of crystalline $\mathrm{SrTiO}_{3}$ nanoparticles. Figure 1(b) shows the line shape of (110) peaks of the ST-550, ST-650, and ST-750 samples. Based on the analysis of XRD spectra, we estimated the mean crystalline size of the nanoparticles as 52, 56, and $69 \mathrm{~nm}$ for the ST-550, ST-650, and ST-750 samples, respectively. The mean crystalline size grew with an increase of annealing temperature. Figure 1(c) shows a cross-sectional transmission electron microscopy (TEM) image of the 200-nm thick ST-550 thin 

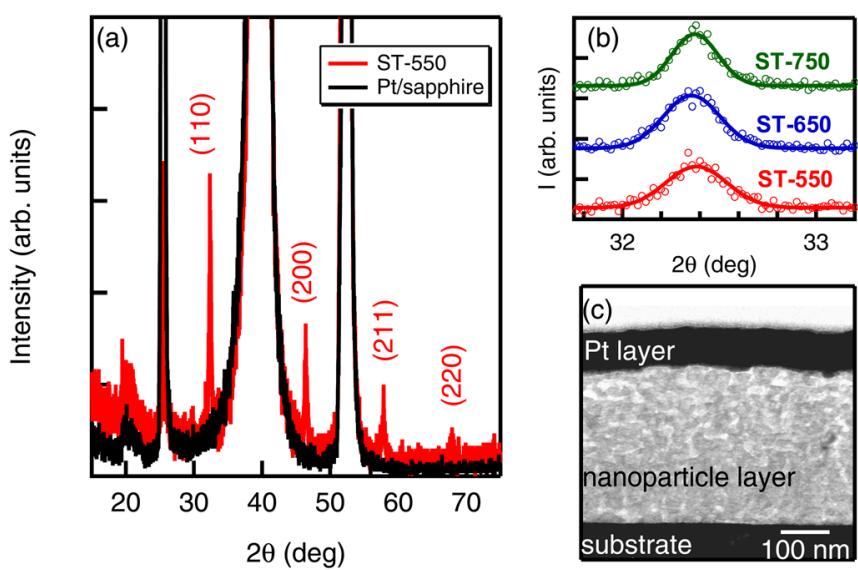

FIG. 1. (Color online) (a) X-ray diffraction patterns of the ST-550 sample and the Pt/sapphire substrate. The Miller indices are assigned to the $\mathrm{x}$-ray diffraction peaks of $\mathrm{SrTiO}_{3}$. The inset shows the line shape of (110) peaks of the ST-550, ST-650, and ST-750 samples. Solid curves are the Lorentzian fitting results. (b) Cross-sectional TEM image of the ST-550 sample.

film. Before thinning process of the sample using a focused ion beam apparatus, the surface of the $\mathrm{SrTiO}_{3}$ nanoparticle thin film was coated with a platinum layer to protect the sample from accelerated ion beams. We observed nanoparticles with several tens of nanometers in the films, supporting the nanoparticle size estimated from $\mathrm{x}$-ray data.

Figure 2 shows time-integrated PL spectra of (a) ST550, (b) ST-650, and (c) ST-750 samples under cw laser excitation (dotted curves) and pulse laser excitation (solid curves). The PL intensities are normalized at their peak intensities, and the PL intensity decays within $10 \mathrm{~ns}$ which is much shorter than the repetition time of pulse laser. Under $\mathrm{cw}$ photoexcitation $\left(2.0 \mathrm{~W} / \mathrm{cm}^{2}\right)$, PL spectra of all samples consist of four broad bands whose peaks are about 1.7, 2.4, 2.9 , and $3.3 \mathrm{eV}$, where the intensity of each PL band depends on the annealing temperature and the monitored position, but the spectral shape of each PL band is insensitive to the monitored position. This indicates the inhomogeneous distribution of defect and impurity densities. On the other hand, under strong pulse laser excitation $\left(425 \mu \mathrm{J} / \mathrm{cm}^{2}\right)$, the broad $2.9-\mathrm{eV}$ PL band is dominantly observed and other low-energy PL bands become relatively weak in all samples. The PL spectrum under pulse laser excitation is insensitive to the monitored position. A similar broad blue PL band is observed in bulk $\mathrm{SrTiO}_{3}$ crystals under strong pulse laser excitation [see the inset of Fig. 3(a)]. It is considered that the 1.7-eV red PL and 2.4-eV green PL bands are related to defects or impurities and the $2.9-\mathrm{eV}$ blue PL reflects an intrinsic carrier recombination process. Hereafter, we discuss the blue PL process of $\mathrm{SrTiO}_{3}$ nanoparticles.

In Fig. 3, we summarize the excitation-density dependences of the PL intensity in the ST-550 sample monitored at 2.9 and $1.7 \mathrm{eV}$ under (a) $\mathrm{cw}$ and (b) pulse laser excitation. Under weak $\mathrm{cw}$ excitation below $0.5 \mathrm{~mW} / \mathrm{cm}^{2}$, both the 2.9 $\mathrm{eV}$ (blue) and 1.7-eV (red) PL intensities show a linear dependence on the excitation density. The blue PL intensity in electron-doped $\mathrm{SrTiO}_{3}$ bulk crystals also linearly increases with the excitation density. ${ }^{8,9}$ Then, we conclude that $\mathrm{SrTiO}_{3}$ nanoparticles contain excess electrons originating from oxygen-deficient surface and that the blue PL originates from

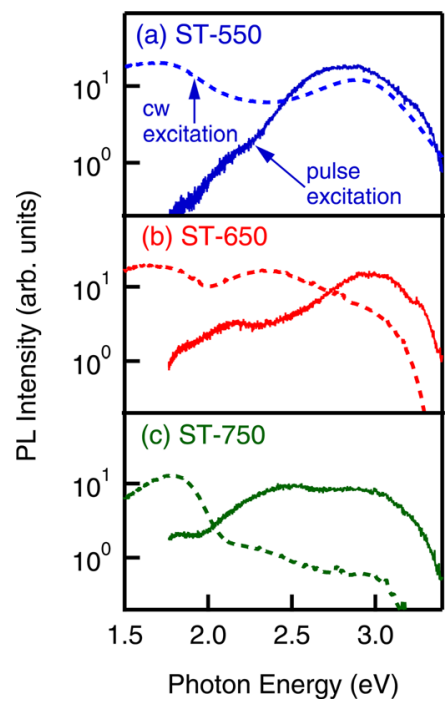

FIG. 2. (Color online) PL spectra of (a) ST-550, (b) ST-650, and (c) ST-750 under $\mathrm{cw}$ laser excitation $\left(2.0 \mathrm{~W} / \mathrm{cm}^{2}\right.$, dotted curves) and pulse laser excitation $\left(425 \mu \mathrm{J} / \mathrm{cm}^{2}\right.$, solid curves). The vertical axis is in logarithmic scale.

the bimolecular (two-body) recombination of excess electrons and photoexcited holes. ${ }^{12}$ Above $0.5 \mathrm{~mW} / \mathrm{cm}^{2}$, the blue PL intensity shows quadratic dependence on the excitation density, suggesting that the blue PL originates from bimolecular recombination of photoexcited electrons and holes. ${ }^{8,9}$

Under pulse laser excitation, the red PL intensity is negligibly small. In Fig. 3(b), with an increase in the excitation density, the blue PL intensity shows a saturation behavior, suggesting the role of nonradiative Auger recombination processes. ${ }^{8,20}$ For $\mathrm{SrTiO}_{3}$ crystals, the carrier recombination dynamics is written by a simple rate equation, which is given by ${ }^{8,9,20,21}$

$$
\begin{gathered}
\mathrm{d} n(t) / \mathrm{d} t=- \\
-A n(t)-B n(t)^{2}-C n(t)^{3}, \\
I_{\mathrm{PL}}(\mathrm{t}) \propto n(\mathrm{t})^{2},
\end{gathered}
$$

where $n(t)$ and $I(\mathrm{t})$ are the carrier density and PL intensity at time $t$, respectively. $A$ represents the single-carrier trapping rate of photocarriers. $C$ represents the intrinsic Auger recombination coefficient involving three carriers such as electron-
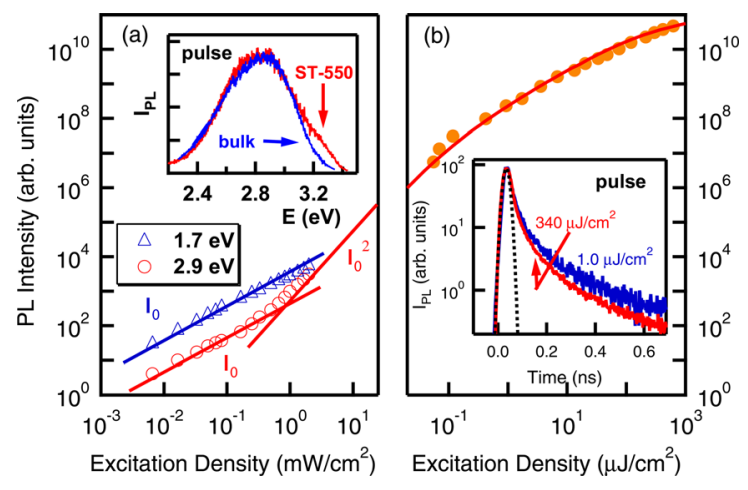

FIG. 3. (Color online) Excitation-density dependence of the PL intensities at $1.7 \mathrm{eV}$ (triangle) and $2.9 \mathrm{eV}$ (circle) under (a) $\mathrm{cw}$ and (b) pulse laser excitation. The inset of (a) shows the PL spectra of the ST-550 and bulk $\mathrm{SrTiO}_{3}$ crystal samples under pulse laser excitation. The solid curve in (b) is the calculated blue PL intensity. The inset of (b) shows the PL decay dynamics at $2.9 \mathrm{eV}$ under 1.0 and $340 \mu \mathrm{J} / \mathrm{cm}^{2}$ excitation. 
electron-hole or electron-hole-hole. Here, note that $B$ consists of two components: radiative recombination and trapassisted Auger recombination. ${ }^{20-22}$ In indirect-gap semiconductor $\mathrm{SrTiO}_{3}$ nanoparticles, the PL efficiency is very low at room temperature and then radiative recombination is negligibly small. Moreover, it is believed that high-density surface states exist due to large surface-to-volume ratios. Thus, the trap-assisted Auger recombination process is significant in nanoparticles, while its rate is negligibly small in $\mathrm{SrTiO}_{3}$ bulk crystals. $^{22}$

To examine the Auger recombination process under strong photoexcitation, we measure the PL decay dynamics. The inset of Fig. 3(b) shows the PL decay dynamics at $2.9 \mathrm{eV}$ of the ST-550 sample under 340 and $1.0 \mu \mathrm{J} / \mathrm{cm}^{2}$ excitation. We also plot the instrumental function in the same figure as a dotted curve. As the excitation density is increased, the PL decays faster and the PL decay curve becomes non-exponential. These behaviors indicate that nonradiative decay rate is enhanced under high-density excitation due to multicarrier interactions and they can be explained by the Auger recombination process, as described by Eqs. (1)(2). The blue PL decay curves in the subnanosecond time region are approximately described by two exponential functions. The decay time of the fast component is several tens of picoseconds, corresponding to Auger recombination rate. The slow decay time is approximately $5 \mathrm{~ns}$, insensitive to the laser excitation intensity and we obtain $A=1.0 \pm 0.1 \times 10^{8} \mathrm{~s}^{-1}$.

We estimate the $B$ and $C$ coefficients of $\mathrm{SrTiO}_{3}$ nanoparticles based on the excitation density dependence of the blue PL intensity. Here, we evaluated the photocarrier density by assuming that the optical absorption coefficient in nanoparticles thin films is the same as that of bulk crystals, because the nanoparticles are closely packed in the thin films as shown in Fig. 1(c). On the basis of Eqs. (1)(2), we fitted the PL intensity as a function of excitation density, where we fixed $A=1 \times 10^{8} \mathrm{~s}^{-1} .{ }^{23}$ The results are plotted in Fig. 3(b) as a solid curve, where the best-fit parameters are $B=6.4 \pm 3.3 \times 10^{-10} \mathrm{~s}^{-1} \mathrm{~cm}^{3}$ and $C=1.8 \pm 0.2 \times 10^{-30}$ $\mathrm{s}^{-1} \mathrm{~cm}^{6}$. The calculated curve well reproduces the experimental results. The $C$ coefficient of nanoparticles is much larger than that of bulk crystals $\left(C=1.3 \times 10^{-32} \mathrm{~cm}^{6} \mathrm{~s}^{-1}\right){ }^{8,9}$

Here, we discuss the origin of the large Auger recombination coefficient of $\mathrm{SrTiO}_{3}$ nanoparticles. Quantum confinement effects such as relaxation of momentum conservation in indirect-gap semiconductors do not critically affect the carrier recombination process, because of large nanoparticle sizes of our samples (about $50 \mathrm{~nm}$ ) and large effective masses of $\mathrm{SrTiO}_{3}$ crystals. Rather, nanoparticles possess large surface-to-volume ratios and the surface plays a crucial role in the enhancement of intrinsic Auger recombination as well as trap-assisted Auger recombination. ${ }^{24-26}$ We can point out that possible enhancement effects are (i) increased final state density and (ii) reduced dielectric constant. In nanoparticles, the final state density of Auger recombination process is anticipated to increase with an increase of surface state density. ${ }^{26,27}$ Moreover, the reduced dielectric constant of $\mathrm{SrTiO}_{3}$ surface should also be considered. It has been reported that the dielectric constants of $\mathrm{SrTiO}_{3}$ nanoparticles and thin films are much smaller than that of bulk crystals. ${ }^{28-}$ 30 The reduction of dielectric constant enhances the Coulomb interaction of electrons and holes, which results in the increase of Auger recombination rate. These two effects enhance the Auger recombination rate. We conclude that surface effects enhance the Auger recombination rate of $\mathrm{SrTiO}_{3}$ nanoparticles.

In conclusion, we studied blue PL spectrum and dynamics of $\mathrm{SrTiO}_{3}$ nanoparticle thin films under strong photoexcitation. The excitation-density dependence of the blue PL intensity and the rapid PL decay indicate Auger-dominated recombination process. We pointed out that the enhancement of the Auger recombination coefficient is related to large surface-to-volume ratios of nanoparticles.

Part of this work was supported by KAKENHI (Grant No. 20104006).

${ }^{1}$ O. N. Tufte and P. W. Chapman, Phys. Rev. 155, 796 (1967).

${ }^{2}$ K. A. Müller and H. Burkard, Phys. Rev. B 19, 3593 (1979).

${ }^{3}$ J. Hemberger, P. Lunkenheimer, R. Viana, R. Böhmer, and A. Loidl, Phys. Rev. B 19, 3593 (1979).

${ }^{4}$ K. van Benthem, C. Elsasser, and R. H. French, J. Appl. Phys. 90, 6156 (2001).

${ }^{5}$ Y. Muraoka, T. Muramatsu, J. Yamaura, and Z. Hiroi, Appl. Phys. Lett. 85, 2950 (2004).

${ }^{6}$ M. Capizzi and A. Frova, Phys. Rev. Lett. 25, 1298 (1970).

${ }^{7}$ Y. Yamada and Y. Kanemitsu, Phys. Rev. B 82, 121103(R) (2010).

${ }^{8}$ H. Yasuda and Y. Kanemitsu, Phys. Rev. B 77, 193202 (2008).

${ }^{9}$ Y. Yamada, H. Yasuda, T. Tayagaki, and Y. Kanemitsu, Phys. Rev. Lett. 102, 247401 (2009).

${ }^{10}$ A. Ohtomo and H. Y. Hwang, Nature (London) 427, 423 (2004).

${ }^{11}$ Y. Kozuka, M. Kim, C. Bell, B. G. Kim, Y. Hikita, and H. Y. Hwang, Nature (London) 462, 487 (2009).

${ }^{12}$ H. Yasuda, Y. Yamada, T. Tayagaki, and Y. Kanemitsu, Phys. Rev. B 78, 233202 (2009).

${ }^{13}$ W. Meevasana, P. D. C. King, R. H. He, S.-K. Mo, M. Hashimoto, A. Tamai, P. Songsiriritthigul, F. Baumberger, and Z.-X. Shenm, Nature Mater. 10, 114 (2011).

${ }^{14}$ A. Brinkman, M. Huijben, M. van Zalk, J. Huijben, U. Zeitler, J. C. Maan, W. G. van der Wiel, G. Rijnders, D. H. A. Blank, and H. Hilgenkamp, Nature Mater. 6, 493 (2007).

${ }^{15}$ N. Reyren, S. Thiel, A. D. Caviglia, L. F. Kourkoutis, G. Hammerl, C. Richter, C. W. Schneider, T. Kopp, A. S. Ruetschi, D. Jaccard, M. Gabay, D. A. Muller, J. M. Triscone, and J. Mannhart, Science 317, 1196 (2007).

${ }^{16}$ Y. Kanemitsu, S. Okamoto, M. Otobe, and S. Oda, Phys. Rev. B 55, R7375 (1997).

${ }^{17}$ S. Okamoto and Y. Kanemitsu, Phys. Rev. B 54, 16421 (1996).

${ }^{18}$ Y. Yamashita, H. Yamamoto, and Y. Sakabe, Jpn. J. Appl. Phys. 43, 6521 (2004).

${ }^{19}$ M. Inoguchi, K. Suzuki, K. Kageyama, H. Takagi, and Y. Sakabe, J. Am. Ceram. Soc. 91, 3850 (2008).

${ }^{20}$ P. T. Landsberg, Recombination in Semiconductors (Cambridge University Press, Cambridge, 1991).

${ }^{21}$ P. T. Landsberg, Appl. Phys. Lett. 50, 745 (1987).

${ }^{22}$ Y. Yamada, H. Yasuda, T. Tayagaki, and Y. Kanemitsu, Appl. Phys. Lett. 95, 121112 (2009).

${ }^{23}$ M. Cardona, Phys. Rev. 140, A651 (1965).

${ }^{24}$ A. Pandey and P. Guyot-Sionnest, J. Chem. Phys. 127, 111104 (2007).

${ }^{25}$ G. E. Cragg and A. L. Efros, Nano Lett. 10, 313 (2010).

${ }^{26}$ S. Taguchi, M. Saruyama, T. Teranishi, and Y. Kanemitsu, Phys. Rev. B 83, 155324 (2011).

${ }^{27}$ A. Cretì, M. Anni, M. Zavelani Rossi, G. Lanzani, L. Manna, and M. Lomascolo, Appl. Phys. Lett. 91, 093106 (2007).

${ }^{28}$ A. A. Sirenko, C. Bernhard, A. Golnik, A. M. Clark, J. Hao, W. Si, and X. X. Xi, Nature (London) 404, 373 (2000).

${ }^{29}$ M. Stengel and N. A. Spaldin, Nature (London) 443, 679 (2006).

${ }^{30}$ M. C. Wang, F. Y. Hsiao, H. H. Huang, and N. C. Wu, Jpn. J. Appl. Phys. 43, 6323 (2004) 